\title{
Myosin Light Chain Kinase Is Not a Regulator of Synaptic Vesicle Trafficking during Repetitive Exocytosis in Cultured Hippocampal Neurons
}

\author{
Hirofumi Tokuoka ${ }^{1}$ and Yukiko Goda ${ }^{1,2}$ \\ ${ }^{1}$ Medical Research Council Laboratory for Molecular Cell Biology and Cell Biology Unit, and ${ }^{2}$ Department of Pharmacology, University College London, \\ London WC1E 6BT, United Kingdom
}

The mechanism by which synaptic vesicles (SVs) are recruited to the release site is poorly understood. One candidate mechanism for trafficking of SVs is the myosin-actin motor system. Myosin activity is modulated by myosin light chain kinase (MLCK), which in turn is activated by calmodulin. $\mathrm{Ca}^{2+}$ signaling in presynaptic terminals, therefore, may serve to regulate SV mobility along actin filaments via MLCK. Previous studies in different types of synapses have supported such a hypothesis. Here, we further investigated the role of MLCK in neurotransmitter release at glutamatergic synapses in cultured hippocampal neurons by examining the effects of two MLCK inhibitors, 1-(5-iodonaphthalene-1-sulfonyl)-1H-hexahydro-1,4-diazepine $\cdot \mathrm{HCl}$ (ML-7) and wortmannin. Bath application of ML-7 enhanced shortterm depression of EPSCs to repetitive stimulation, whereas it reduced presynaptic release probability. However, ML-7 also inhibited action potential amplitude and voltage-gated $\mathrm{Ca}^{2+}$ channel currents. These effects were not mimicked by wortmannin, suggesting that ML-7 was not specific to MLCK in hippocampal neurons. When SV exocytosis was directly triggered by a Ca ${ }^{2+}$ ionophore, calcimycin, to bypass voltage-gated $\mathrm{Ca}^{2+}$ channels, ML-7 had no effect on neurotransmitter release. Furthermore, when SV exocytosis elicited by electrical field stimulation was monitored by styryl dye, FM1-43 [N-(3-triethylammoniumpropyl)-4-(4-(dibutylamino)styryl) pyridinium dibromide], the unloading kinetics of the dye was not altered in the presence of wortmannin. These data indicate that MLCK is not a major regulator of presynaptic SV trafficking during repetitive exocytosis at hippocampal synapses.

Key words: synaptic vesicle recycling; exocytosis; myosin light chain kinase; ML-7; wortmannin; repetitive stimulation

\section{Introduction}

Whereas the machinery for synaptic vesicle (SV) exocytosis has been extensively studied, how vesicles are recruited to the readily releasable pool (RRP) has remained elusive. Intracellularly, vesicles move by either passive diffusion or active transport mediated by motor proteins. Of these, active transport by the myosin-actin system has been proposed to direct the SV traffic within the nerve terminal where microtubules are generally scarce (Langford, 1995). Myosin family proteins are actin-based motor ATPases that regulate various cellular functions from muscle contraction, cell motility to intracellular organelle traffic (Berg et al., 2001; De La Cruz and Ostap, 2004). Myosin activity is modulated by phosphorylation of its regulatory light chain by myosin light chain kinase (MLCK) (Kamm and Stull, 2001). Because MLCK is activated by calmodulin, a rise in presynaptic $\mathrm{Ca}^{2+}$ triggered by an intense stimulus of the sort that depletes the RRP, may enhance

Received Aug. 7, 2006; revised Sept. 28, 2006; accepted Sept. 28, 2006.

This work was supported by the Medical Research Council, National Institutes of Health Grant R01MH66676, and the European Commission Framework VI (EUSynapse Project LSHM-CT-2005-019055). H.T. was a recipient of the postdoctoral fellowship for research abroad from the Japan Society for the Promotion of Science.

Correspondence should be addressed to Yukiko Goda, Medical Research Council Laboratory for Molecular Cell Biology and Cell Biology Unit, University College London, Gower Street, London WC1E 6BT, UK. E-mail: y.goda@ucl.ac.uk.

DOI:10.1523/JNEUROSCI.3400-06.2006

Copyright $\odot 2006$ Society for Neuroscience $\quad$ 0270-6474/06/2611606-09\$15.00/0 myosin motor activity through MLCK to promote RRP refilling. The reported facilitation of RRP replenishment by the rise in intracellular $\mathrm{Ca}^{2+}$ (Stevens and Wesseling, 1998) is consistent with such $\mathrm{Ca}^{2+}$-dependent augmentation of SV mobilization via MLCK activation.

Several reports to date support the hypothesis that myosin and MLCK regulate SV mobility. Actin and myosin are localized to presynaptic sites (Drenckhahn and Kaiser, 1983; Dunaevsky and Connor, 2000; Morales et al., 2000). In sympathetic neurons, inhibiting myosin II decreases synaptic transmission (Mochida et al., 1994; Takagishi et al., 2005), whereas in hippocampal neurons, MLCK inhibitors, 1-(5-iodonaphthalene-1-sulfonyl)- $1 H$-hexahydro-1,4-diazepine $\cdot \mathrm{HCl}$ (ML-7) and 1-(5-chloronaphthalene-1sulfonyl)-1 $H$-hexahydro-1,4-diazepine $\mathrm{HCl}$ (ML-9), reduce the access to the reserve SV pool during repetitive stimulation (Ryan, 1997). Additionally, ML-7 decreases the intraterminal mobility of SVs under basal conditions (Jordan et al., 2005). Nevertheless, lack of effects of actin and myosin disruption reported by some suggests that MLCK may not participate in trafficking of SVs to the releasable pool at small CNS synapses. The rates of RRP replenishment and repriming of newly endocytosed SVs remain unchanged on disrupting F-actin (Morales et al., 2000; Sankaranarayanan et al., 2003), and in myosin Va mutant mice, shortterm plasticity and mobilization of the reserve SV pool are normal (Schnell and Nicoll, 2001). Thus, it has remained unclear 
whether myosin and MLCK in general, play a key role in mobilizing SVs to the releasable pool in small presynaptic boutons.

Here, we further investigated the role of MLCK in synaptic transmission in cultured hippocampal neurons using MLCK inhibitors, ML-7 and wortmannin. ML-7, the more specific of the two inhibitors, reduced presynaptic release probability and enhanced short-term depression, consistent with the previous analysis of ML-7 on SV exocytosis. However, additional experiments revealed that the effects of ML-7 resulted from nonspecific inhibition of neuronal excitability and $\mathrm{Ca}^{2+}$ channel currents rather than by inhibiting MLCK. ML-7 did not block the replenishment of the RRP and neurotransmitter release evoked by calcimycin, a $\mathrm{Ca}^{2+}$ ionophore. Furthermore, wortmannin did not slow the $\mathrm{N}$-(3-triethylammoniumpropyl)-4-(4-(dibutylamino)styryl) pyridinium dibromide (FM1-43) unloading kinetics during repetitive field stimulation. These data suggest that MLCK is not a major regulator of SV mobility, and question the idea that the modulation of myosin activity by MLCK plays a role in SV trafficking in small CNS synapses.

\section{Materials and Methods}

Cell culture. Dissociated cultures of hippocampal CA1-CA3 neurons were prepared from postnatal day 0 rats as described previously with minor modifications (Morales et al., 2000). Coverslips were coated by spraying $4 \mathrm{~mm}$ acetic acid solution containing $5 \mu \mathrm{g} / \mathrm{ml}$ poly-D-lysine (BD Biosciences, San Jose, CA) and $0.4 \mathrm{mg} / \mathrm{ml}$ rat tail collagen. Astrocytes were preplated with culture media consisting of BME (Invitrogen, San Diego, CA), 10\% fetal calf serum (BD Biosciences), $1 \times$ Glutamax (Invitrogen), $20 \mathrm{~mm}$ D-glucose, and penicillin/streptomycin (BD Biosciences). After 3-7 d, dissociated neurons were plated on astrocytes. At 1 or $2 \mathrm{~d}$ in vitro (DIV), media were exchanged to Neurobasal medium (Invitrogen) containing B-27 (Invitrogen), $0.5 \mathrm{~mm}$ glutamine, $25 \mu \mathrm{M}$ $\beta_{2}$-mercaptoethanol, $2 \%$ fetal calf serum, and penicillin/streptomycin. In some experiments (see Figs. 2, 5), autaptic cultures were used (Bekkers and Stevens, 1991; Morales et al., 2000). In this case, coverslips were coated with $0.15 \%$ agarose before spraying poly-D-lysine/collagen. For immunocytochemistry, neurons were directly plated on coverslips coated with $0.2 \%$ polyethylenimine (Sigma, St. Louis, MO) and $10 \mu \mathrm{g} / \mathrm{ml}$ laminin (Sigma), and $4 \mu \mathrm{M}$ cytosine-arabinoside (Sigma) was added to culture media at 2 DIV. Neurons were used for experiments at 13-18 DIV. Animal care and use were approved by the United Kingdom Home Office.

Immunocyotochemistry. Immunocytochemistry was performed as described previously (Morales et al., 2000). Primary antibodies were antiMLCK polyclonal antibody (Covance, Princeton, NJ), anti-neurofilament $\mathrm{H}$ subunit monoclonal antibody (clone RMO-24; Millipore, Bedford, MA) and anti-synaptotagmin I monoclonal antibody (Y. Goda Laboratory). Secondary antibodies were Cy2-conjugated anti-sheep IgG and Texas Red-conjugated anti-mouse IgG (Jackson ImmunoResearch Laboratories, West Grove, PA). Fluorescence images were captured with a cooled CCD camera (CoolSNAP fx; Photometrics, Tucson, AZ) attached to a BX50WI microscope (Olympus, Tokyo, Japan) with $100 \times$ oil objective lens [1.45 numerical aperture (NA); Olympus] and MetaMorph software (Universal Imaging Corporation, West Chester, PA).

Electrophysiology. Whole-cell patch-clamp recordings were performed using pipette solution containing the following (in mM): $124 \mathrm{~K}$ gluconate, $16 \mathrm{KCl}, 5 \mathrm{NaCl}, 3 \mathrm{MgCl}_{2}, 5$ HEPES, pH 7.2, 0.2 EGTA, 2 ATP, 10 phosphocreatine, $0.5 \mathrm{GTP}$, and $25 \mathrm{U} / \mathrm{ml}$ phosphocreatine kinase at 295 mOsm. In some experiments, perforated patch recording was performed using pipette solution containing $0.3 \mathrm{mg} / \mathrm{ml}$ amphotericin B deoxycholate. Electrode resistance was $2-5 \mathrm{M} \Omega$. Series resistance was $<20 \mathrm{M} \Omega$, and compensated at $70-80 \%$. External bath solution contained the following (in mM): $137 \mathrm{NaCl}, 5 \mathrm{KCl}, 10$ D-glucose, 5 HEPES, pH 7.3, 0.1 picrotoxin, $2 \mathrm{CaCl}_{2}, 2 \mathrm{MgCl}_{2}, 0.05 \mathrm{APV}, 0.001 \mathrm{CNQX}$ (to avoid saturation of AMPA receptors and help achieve a better V-clamp), at 295 mOsm.

For voltage-clamp recordings, holding potential was set at $-70 \mathrm{mV}$, and autaptic EPSC was elicited by a $1 \mathrm{~ms}$ depolarizing square voltage pulse to $0 \mathrm{mV}$. For measurement of EPSC amplitude, mean current between -2 to -1 ms just before each stimulus was used as the baseline. Total charge transfer, synchronous release, and asynchronous release during $20 \mathrm{~Hz}$ repetitive stimulation were analyzed as described previously (Otsu et al., 2004), with time window of 3-49 ms after each stimulus. RRP size was measured by perfusing hypertonic external bath solution containing $0.5 \mathrm{~m}$ sucrose for $4 \mathrm{~s}$. For calcimycin experiments, CNQX was omitted from external bath solution. For current-clamp recordings, $10 \mu \mathrm{M} C N Q X$ and $1 \mathrm{~mm} \mathrm{CdCl}_{2}$ were added to the external bath solution, and current was adjusted to maintain a resting potential near $-70 \mathrm{mV}$.

For calcium channel current recording, the external bath solution contained the following (in mM): $117 \mathrm{NaCl}, 5 \mathrm{BaCl}_{2}, 10 \mathrm{CsCl}, 10$ tetraethylammonium (TEA)-Cl, $1 \mathrm{MgCl}_{2}, 10$ HEPES, pH 7.3, 10 D-glucose, 0.001 TTX, 0.01 CNQX, 0.1 picrotoxin, at $295 \mathrm{mOsm}$. Pipette solution contained the following (in mM): $110 \mathrm{CsCl}, 5$ TEA-Cl, $3 \mathrm{MgCl}_{2}, 10$ EGTA, 20 HEPES, pH 7.2, 2 ATP, 10 phosphocreatine, $0.5 \mathrm{GTP}$, and $25 \mathrm{U} / \mathrm{ml}$ phosphocreatine kinase, at $295 \mathrm{mOsm}$. Holding potential was set at -80 $\mathrm{mV}$. Current size was normalized by cell capacitance to correct for the variability in cell size.

ML-7 (10 $\mu \mathrm{M}$; Calbiochem, La Jolla, CA), wortmannin (10 $\mu \mathrm{M}$; Tocris Bioscience, Bristol, UK), or DMSO (0.1\%, control) was perfused during recording, or neurons were pretreated for $15 \mathrm{~min}$ before recording. All experiments were performed at room temperature.

FM1-43 experiments. Coverslips were placed in a field stimulation chamber in which a pair of platinum wire electrodes was positioned $1 \mathrm{~cm}$ apart. Action potential (AP) was evoked by a $2 \mathrm{~ms}, 20 \mathrm{~V}$ square pulse. External bath solution was the same as that used for the whole-cell recording except $10 \mu \mathrm{M}$ CNQX and $50 \mu \mathrm{M}$ APV were added. Neurons were loaded with FM1-43 (10 $\mu \mathrm{M}$; Invitrogen) by $900 \mathrm{APs}$ at $10 \mathrm{~Hz}$. After loading, neurons were quickly washed with $1 \mathrm{~mm}$ Advasep-7 (Invitrogen) for $1 \mathrm{~min}$, and treated with $0.1 \%$ DMSO or $10 \mu \mathrm{M}$ wortmannin for 15 min. FM1-43 was unloaded by $900 \mathrm{APs}$ at $20 \mathrm{~Hz}$. Images were acquired at $0.5 \mathrm{~Hz}, 300 \mathrm{~ms}$ per frame, with a $60 \times$ water immersion lens (NA, 0.9; Olympus), $470 / 50 \mathrm{~nm}$ excitation filter, 500LP dichroic mirror, and $545 / 75 \mathrm{~nm}$ emission filter, using the same setup as described above for immunofluorescence experiments. Region of interest size was set to 2 . Neutral density filters were engaged to reduce the intensity of excitation light to $6.25 \%$. Images were analyzed with NIH Image J. To quantify FM1-43 unloading rate, fluorescence intensity at the central $4 \times 4$ pixel box of each puncta was measured. Background signal from regions free of neuronal processes was subtracted. Data were normalized to the average fluorescence of first six frames during the $10 \mathrm{~s}$ period before field stimulation.

Statistics. Paired or nonpaired $t$ test, Kolmogorov-Smirnov test, Dunnett's multiple comparison test, one-way ANOVA, or repeated-measures ANOVA were used as required. When interaction was significant in repeated-measures ANOVA, all groups were compared at each $x$-axis point by $t$ test or Dunnett's multiple comparison test. A value of $p<0.05$ was taken as significant. Data are shown as mean \pm SEM.

\section{Results}

\section{Localization of MLCK in axons}

We first examined the expression pattern of MLCK in cultured hippocampal neurons by immunocytochemistry. MLCK was broadly localized to neuronal soma and neurites, and prominent signal was observed in axons as identified by the labeling for phosphorylated neurofilament $\mathrm{H}$ subunit (Benson et al., 1996) (Fig. 1A). Double labeling of MLCK with a presynaptic marker, synaptotagmin, however, showed only partially overlapping signals (Fig. $1 B, C$ ). These data suggest that, although MLCK is distributed to axons, it is not enriched at presynaptic terminals.

\section{The effect of ML-7 on release: changes in release probability and short-term depression}

Previous fluorescence imaging studies indicated that ML-7 and its structural homolog, ML-9, inhibited the mobility of FM1-43- 
labeled SVs in presynaptic terminals of hippocampal neurons (Ryan, 1997; Jordan et al., 2005). We therefore examined the effect of ML-7, which exhibited an order of magnitude smaller $K_{\mathrm{i}}$ value for MLCK than ML-9 (Saitoh et al., 1987), on synaptic transmission by performing whole-cell patch-clamp recordings in autaptic hippocampal culture system. Bath perfusion of $10 \mu \mathrm{M} \mathrm{ML}-7$ significantly reduced the autaptic EPSC amplitude within $15 \mathrm{~min}$ of its application ( $t$ test, $p<0.01)$ (Fig. $2 A$ ), whereas DMSO control $(0.1 \%)$ had no significant effect ( $t$ test, $p>0.2$ ) (Fig. $2 B$ ). Summary of all experiments show that the mean EPSC amplitudes after ML-7 and DMSO application were $47 \pm 9$ and $94 \pm$ $5 \%$, respectively, of baseline values ( $t$ test, $p<0.001$ ) (Fig. 2C). Therefore, ML-7 changes basal synaptic transmission.

Because EPSCs were monitored throughout the application of ML-7, inhibition of EPSC by ML-7 could be use dependent. Moreover, ML-7 was shown to reduce FM1-43 dye unloading during repetitive stimulation (Ryan, 1997). We thus tested the effect of ML-7 on repetitive synaptic transmission electrophysiologically. In cultured hippocampal neurons, a $20 \mathrm{~Hz}$ stimulus train promotes short-term depression of EPSC amplitude (Hagler and Goda, 2001). Application of ML-7 significantly enhanced the short-term depression in the same neurons (repeatedmeasures ANOVA, interaction, $p<0.01$ ) (Fig. 2D). Neurotransmitter release consists of temporally distinct synchronous and asynchronous release components, in which the latter predominates at later pulse trains when $\mathrm{Ca}^{2+}$ accumulates in the presynaptic terminal. Analysis of synchronous and asynchronous components to individual pulses during successive stimulation indicated that ML-7 reduced both components of release, thereby inhibiting the total charge transfer of individual synaptic responses (repeated-measures ANOVA, interaction, $p<0.001$ in all cases) (Fig. $2 E-G)$. DMSO (0.1\%) had no effect on short-term depression (supplemental Fig. 1 $A, B$, available at www.jneurosci. org as supplemental material).

The enhanced short-term depression could result from faster depletion of the RRP either through an increase in the vesicular release probability or a decrease in the ability to fill the RRP. Alternatively, the release probability could be progressively reduced by a release-independent mechanism during successive stimulation. To determine the effect of ML-7 on vesicular release probability, we compared the total charge transfer of EPSCs induced by a single AP to the size of RRP estimated by the perfusion of hypertonic sucrose solution (Rosenmund and Stevens, 1996). Pretreatment of neurons with ML-7 significantly reduced the total charge transfer of EPSC by $47 \%$ ( $t$ test, $p<0.05$ ) (Fig. $2 H$ ) as expected from the reduction in EPSC amplitude, whereas RRP size was not altered ( $t$ test, $p>0.7$ ) (Fig. $2 I$ ). Thus, ML-7 reduces the vesicular release probability ( $t$ test, $p<0.05$ ) (Fig. $2 J$ ). The basis for the observed enhancement of short-term depression by ML-7 could be attributable to inefficient recruitment of vesicles to RRP as suggested previously, and/or progressive decline in release probability by a mechanism independent of exocytosis and depletion of RRP.

We also examined the effect of ML-7 on quantal AMPA receptor responses. Unlike the EPSC, the amplitude of miniature EPSC (mEPSC) was not reduced but slightly increased (before ML-7, $25.9 \pm 0.4 \mathrm{pA}$; after ML-7, $29.7 \pm 0.4 \mathrm{pA}$; Kolmogorov-Smirnov test, $p<0.01$ ) (supplemental Fig. $2 C$, available at www. jneurosci.org as supplemental material). Unexpectedly, the mEPSC frequency was elevated by approximately fivefold in the presence of ML-7 (supplemental Fig. 2A,B, available at www. jneurosci.org as supplemental material), and similar results were seen in two other independent experiments. Overlapping of the mEPSC waveforms by increased mEPSC frequency thus likely contributed to the observed small increase in mEPSC amplitude. A lack of reduction of quantal amplitude by ML-7 argues against the possibility that a decrease in EPSC amplitude (Fig. 2) is attributable to postsynaptic reduction in the number of functional AMPA receptors.

To test the specificity of the observed increase in mEPSC frequency by ML-7, we monitored the effect of wortmannin on mEPSCs. Wortmannin is another pharmacological inhibitor of MLCK that blocks phosphatidylinositol 3-kinase (PI3K) activity at a lower concentration $\left(K_{\mathrm{i}}, 3 \mathrm{nM}\right.$ ) (Arcaro and Wymann, 1993; Yano et al., 1995) and MLCK at a higher concentration $\left(K_{\mathrm{i}}, 300\right.$ nM) (Nakanishi et al., 1992; Takayama et al., 1996; Sakurada et al., 1998; Qi et al., 2002; Grimm et al., 2005). Bath application of wortmannin $(10 \mu \mathrm{M})$ at a concentration that is sufficient to block both PI3K and MLCK, changed neither mEPSC frequency (supplemental Fig. $2 D$, available at www.jneurosci.org as supplemen- 
A

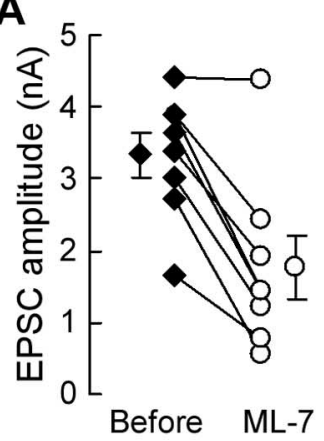

B

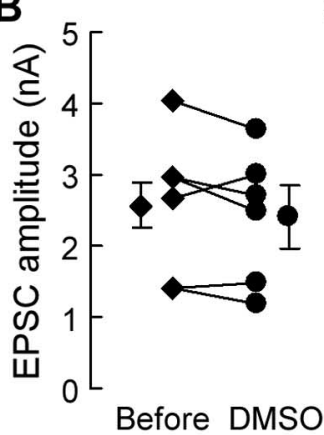

C
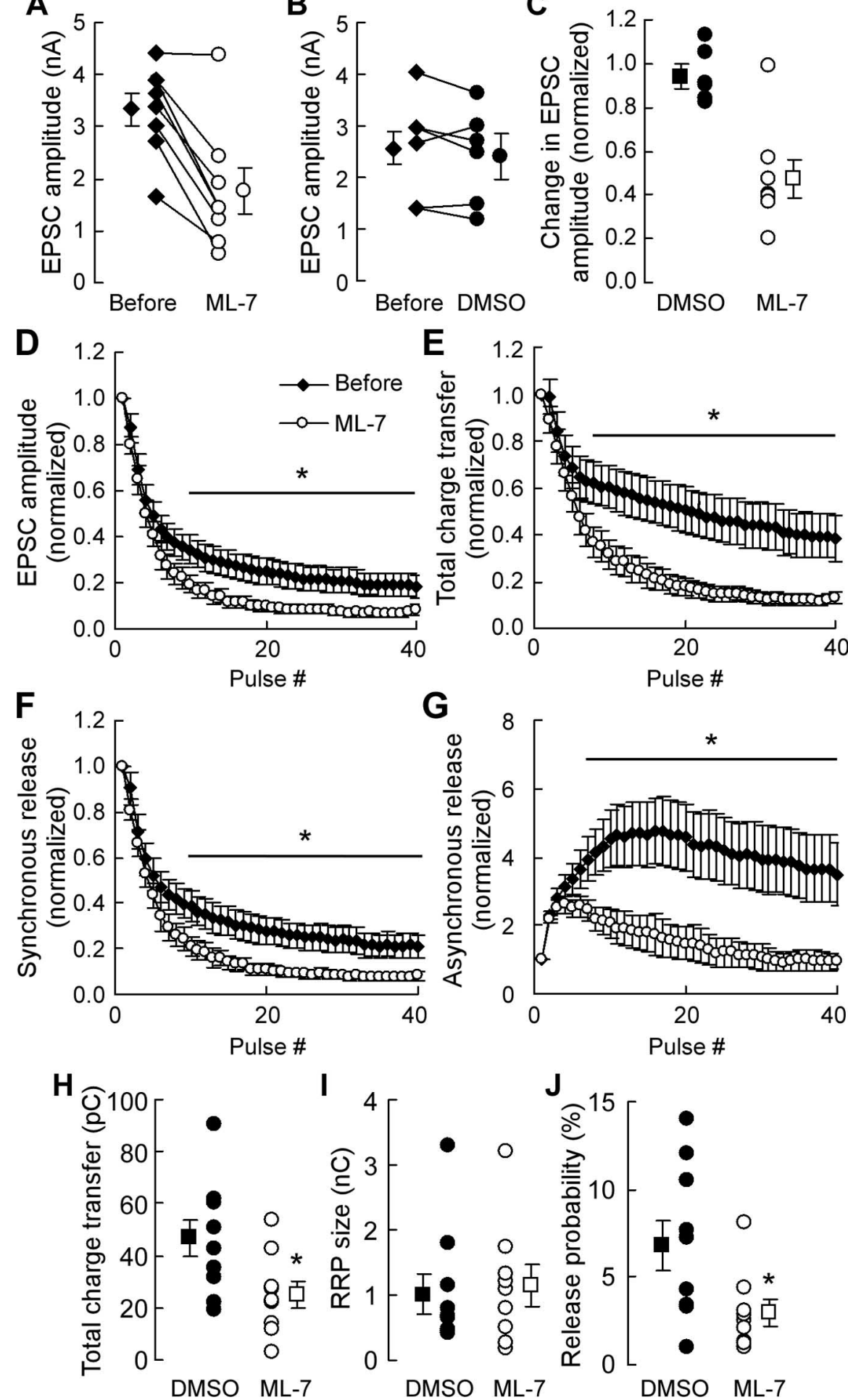

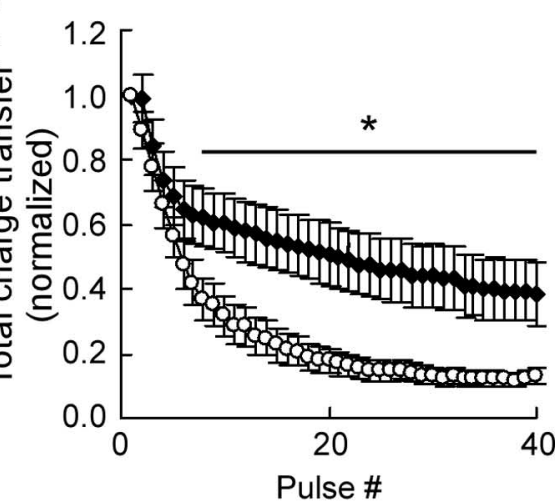

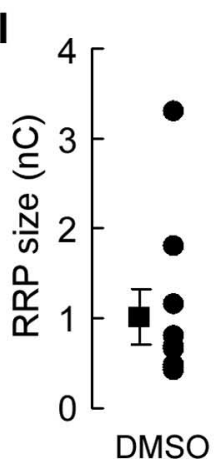
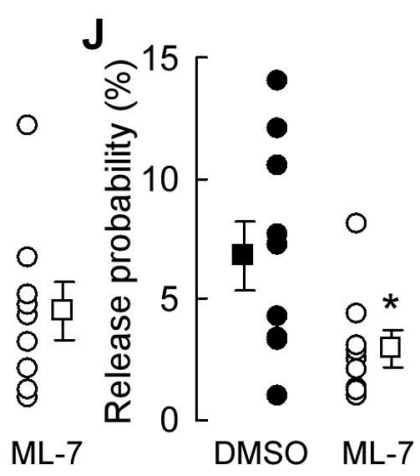

Figure 2. ML-7 reduces release probability but enhances short-term depression. $A-C$, Effect of ML-7 on EPSC amplitude. Mean EPSC amplitude in autaptic neurons is shown before and during perfusion of $10 \mu \mathrm{MML}-7(\boldsymbol{A} ; n=8)$ or control $0.1 \% \mathrm{DMSO}(\boldsymbol{B} ; n=$ 6), and the extent of change in EPSC amplitude is summarized in C (square symbols). $\boldsymbol{D}-\mathbf{G}$, Effect of ML-7 on short-term depression. Neurons were stimulated by a train of 40 pulses at $20 \mathrm{~Hz}$ before and after ML-7 perfusion $(n=8)$. Short-term depression of EPSC amplitude $(\boldsymbol{D})$, total charge transfer $(\boldsymbol{E})$, synchronous release $(\boldsymbol{F})$, and asynchronous release $(\boldsymbol{G})$ are shown. Data are normalized to the first response of the train. ML-7 reduced all four parameters examined ( ${ }^{*} p<0.05 ; t$ test). $\boldsymbol{H}-\boldsymbol{J}$, Estimation of RRP size and release probability. Neurons were pretreated with $10 \mu \mathrm{M} \mathrm{ML-7}(n=10)$ or control $0.1 \%$ DMSO $(n=9)$ for $15 \mathrm{~min}$, followed by patch-clamp recording. Mean total charge transfer elicited by a single AP-evoked release $(\boldsymbol{H})$ and the estimate of RRP size by a $4 \mathrm{~s}$ perfusion of $0.5 \mathrm{~m}$ sucrose $(\boldsymbol{I})$ are shown for each condition. These values were used to estimate release probability defined as follows: [charge transferred by single AP-induced transmission]/[charge of RRP] ( $J$ ). Square symbols denote the mean values for each measurement. ML-7 treatment decreases the response elicited by a single AP and the release probability $\left({ }^{*} p<\right.$ $0.05 ; t$ test), whereas the RRP size is unchanged ( $p>0.2 ; t$ test). Error bars indicate SEM.

tal material) nor amplitude (data not shown). Therefore, ML-7 most likely increases mEPSC frequency by a mechanism independent of MLCK. Note that the wortmannin we used was active, because $10 \mu \mathrm{M}$ wortmannin, by $30 \mathrm{~min}$ of incubation, visibly changed astrocyte morphology and actin filament organization, which were not observed with control DMSO (supplemental Fig. 6, available at www.jneurosci.org as supplemental material).

\section{ML-7 changes AP amplitude and voltage-gated $\mathrm{Ca}^{2+}$ channel current nonspecifically}

The reduced vesicular release probability and enhanced short-term depression by ML-7 raised the possibility that ML-7 could affect AP generation and amplitude (Brody and Yue, 2000). To test the effect of ML-7 on the excitability of neurons, we performed current-clamp recordings. Input resistance was not altered by ML-7 (Fig. 3A), indicating a lack of effect on passive channel currents. We then compared the number of APs evoked by a $100 \mathrm{~ms}$ current injection between control and ML-7-treated neurons. ML-7 significantly reduced the number of APs elicited relative to control DMSO (repeated-measures ANOVA, interaction, $p<0.0001$ ) (Fig. $3 B, C)$. Because ML-7 exerted a nonspecific effect on mEPSC frequency that was not reproduced by wortmannin, we tested whether the attenuation of APs by ML-7 could be mimicked by wortmannin. Application of $10 \mu \mathrm{M}$ wortmannin did not alter the number of spikes evoked by current injection (Fig. $3 A, C$ ). The inhibitory effect of ML-7 on APs, therefore, was independent of MLCK.

To determine whether attenuation of APs contributed to the enhancement of short-term depression by ML-7, we directly monitored AP amplitudes elicited by repetitive stimulation in the absence or presence of ML-7. Injection of short current pulses at $20 \mathrm{~Hz}$ in control neurons triggered a train of APs whose amplitudes showed a slight progressive decrease (Fig. $3 D, E)$. However, in neurons pretreated with ML-7, a pronounced depression of AP amplitudes occurred (repeatedmeasures ANOVA, interaction, $p<$ 0.0001) (Fig. 3E). Moreover, ML-7 decreased the amplitude and increased the half-maximal width of the AP waveform to the first stimulus pulse (Dunnett's multiple comparison test, $p<0.05,0.01$, for AP amplitude and width, respectively) (Fig. $3 F, G)$. These changes in AP amplitude and shape were not observed by $10 \mu \mathrm{M}$ 
wortmannin (Fig. 3E-G), indicating that nonspecific effects of ML-7 extend to neuronal excitability. Lower concentration of wortmannin $(0.1 \mu \mathrm{M})$, under conditions inhibitory to PI3K but not to MLCK, also did not affect AP amplitude (supplemental Fig. 3, available at www.jneurosci.org as supplemental material). Notably, we found that ML-9, the structural homolog of ML-7, modified APs similarly to ML-7 (supplemental Fig. 3, available at www. jneurosci.org as supplemental material).

The observed attenuation of AP amplitude by ML-7 provides an explanation for the reduction of vesicular release probability. Because a reduction in voltage-gated $\mathrm{Ca}^{2+}$ channel (VGCC) currents could also contribute to the reduced release probability and enhanced synaptic depression (Xu and $\mathrm{Wu}, 2005)$, we next performed somatic recordings of VGCC currents. ML-7 significantly reduced the somatic $\mathrm{Ca}^{2+}$ current compared with control DMSO (repeated-measures ANOVA, interaction, $p<0.001$ ) (Fig. 4A, B), whereas cell capacitance was unchanged (data not shown). ML-9 also reduced the somatic VGCC current (supplemental Fig. 4, available at www. jneurosci.org as supplemental material). In contrast, $10 \mu \mathrm{M}$ wortmannin exerted no effects on the $\mathrm{Ca}^{2+}$ currents relative to control (Fig. 4B). Therefore, ML-7 (and ML-9) nonspecifically inhibits not only APs but also VGCC currents, and these nonspecific effects are likely to be the major cause of the observed reduction in EPSC amplitude and enhancement of short-term depression by ML-7. Furthermore, these findings raise questions about the interpretation of previously reported inhibitory effects of ML-7 and ML-9 on presynaptic vesicle traffic. To clarify the potential role of MLCK in regulating neurotransmitter release, we turned to assays of neurotransmitter release that do not rely on the activities of nonspecific targets of ML-7 reported thus far.

\section{ML-7 does not affect calcimycin- induced release and $R R P$ recovery after depletion}

To avoid the side effects of ML-7 on APs and VGCCs, we examined neurotransmitter release induced by calcimycin, a $\mathrm{Ca}^{2+}$ ionophore. Calcimycin-induced release bypasses the AP-triggered, depolarizationcoupled $\mathrm{Ca}^{2+}$ influx, and therefore it allows for a direct study of the efficacy of SV fusion machinery downstream of $\mathrm{Ca}^{2+}$ influx into the presynaptic boutons (Fernandez-Chacon et al., 2001). When the kinetics of calcimycin-induced neurotransmitter release was compared between control and ML-7-treated neurons, no significant difference was found (repeated-measures ANOVA, interaction, $p=1$;

A

B
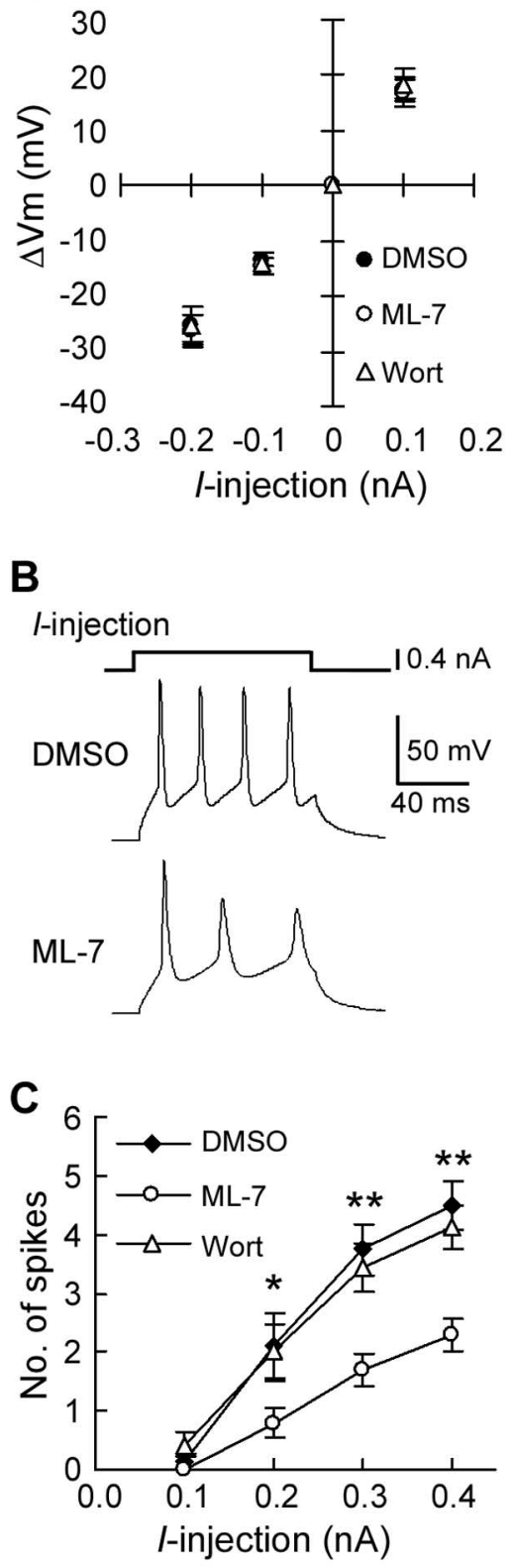

I-injection

ШШШШШШШШШШШШШШШШ__ 2 nA

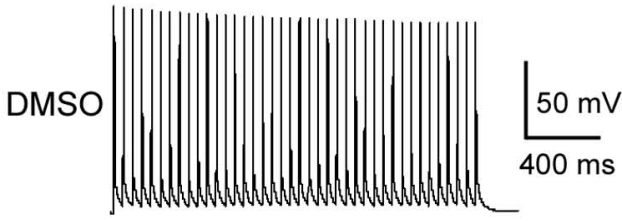

ML-7

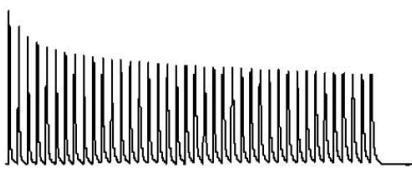

E
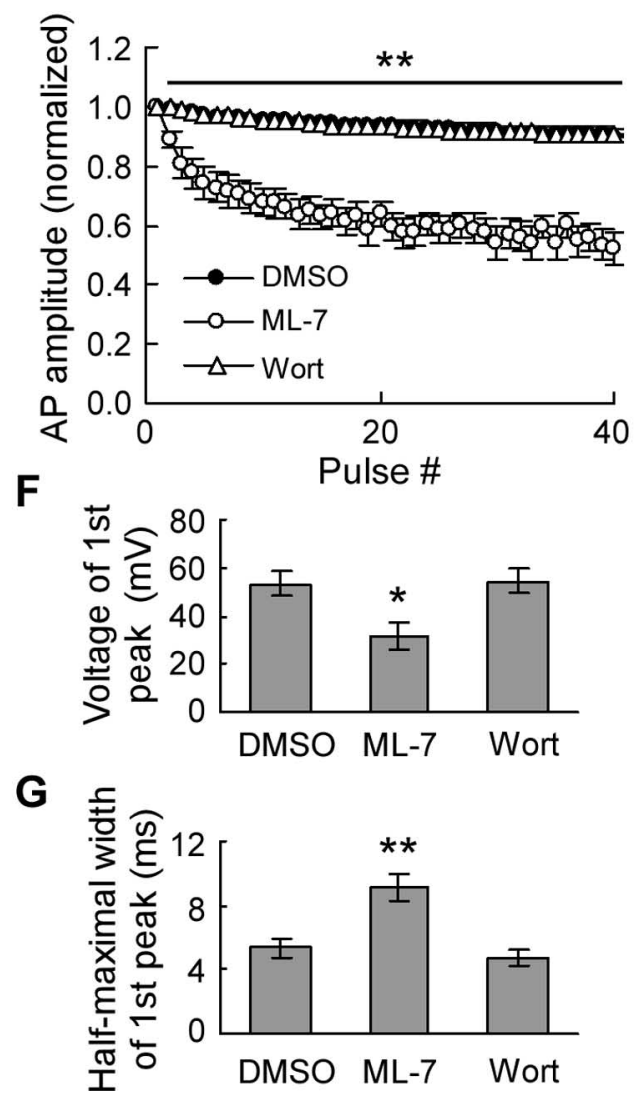

Figure 3. ML-7 reduces the excitability of neurons independently of MLCK activity. $A, A$ plot of $I-V$ relationship in neurons pretreated with $0.1 \%$ DMSO $(n=7), 10 \mu \mathrm{MML}-7(n=8)$, or $10 \mu \mathrm{m}$ wortmannin $(n=6)$. $\boldsymbol{B}$, Representative traces of APs elicited by current injection $(0.4 \mathrm{nA} ; 100 \mathrm{~ms})$ in neurons pretreated with DMSO or ML-7. C, Number of AP spikes generated during current injection in neurons pretreated with DMSO $(n=8), \mathrm{ML}-7(n=10)$, or wortmannin $(n=7)$. The number of APs is significantly reduced in the presence of ML-7 but not in wortmannin $\left({ }^{*} p<0.05,{ }^{* *} p<0.01\right.$; Dunnett's test, DMSO vs ML-7). D, Representative traces of APs induced by repetitive $2 \mathrm{~ms}, 2 \mathrm{nA}$ current injections at $20 \mathrm{~Hz}$ in neurons pretreated with DMSO or ML-7. E, Summary of the peak AP amplitude change during repetitive stimulation. Data are normalized to the first peak. AP amplitude is substantially decreased in the presence of ML-7 $(n=7)$, whereas wortmannin $(n=7)$ is without effect relative to DMSO control $(n=9)$ $\left({ }^{* *} p<0.01\right.$; Dunnett's test, DMSO vs ML-7). $\boldsymbol{F}, \mathbf{G}$, Summary of the peak amplitude and half-maximal width of the first AP. ML-7 decreases the peak amplitude $\left({ }^{*} p<0.05\right.$; Dunnett's test, DMSO vs ML-7) and increases half-maximal width of the first AP $\left({ }^{* *} p<\right.$ 0.01; Dunnett's test, DMSO vs ML-7). Error bars indicate SEM.

DMSO vs ML-7, $p>0.7$ ) (Fig. $5 A, B$ ). Cumulative charge transfer was also not different between control and ML-7-treated neurons (repeated-measures ANOVA, interaction, $p=1$; DMSO vs ML-7, $p>0.8$ ) (Fig. 5C). A lack of change in release by stimulation that bypasses the depolarization-coupled $\mathrm{Ca}^{2+}$ influx is consistent with a lack of effect of ML-7 on sucrose-induced release of 
A

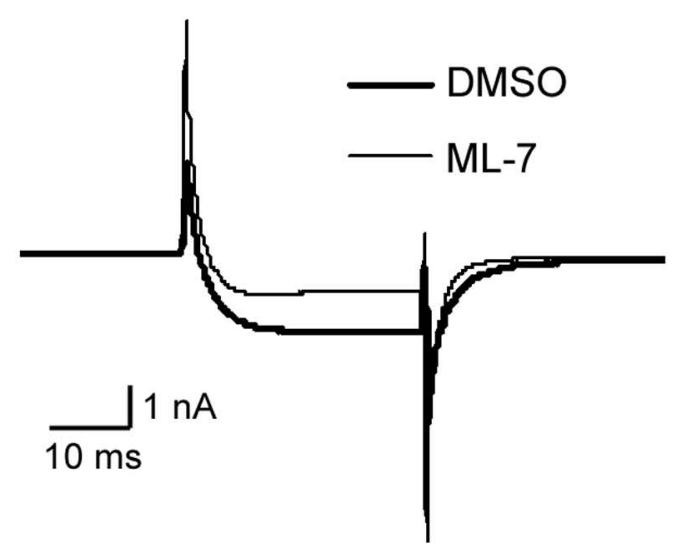

B

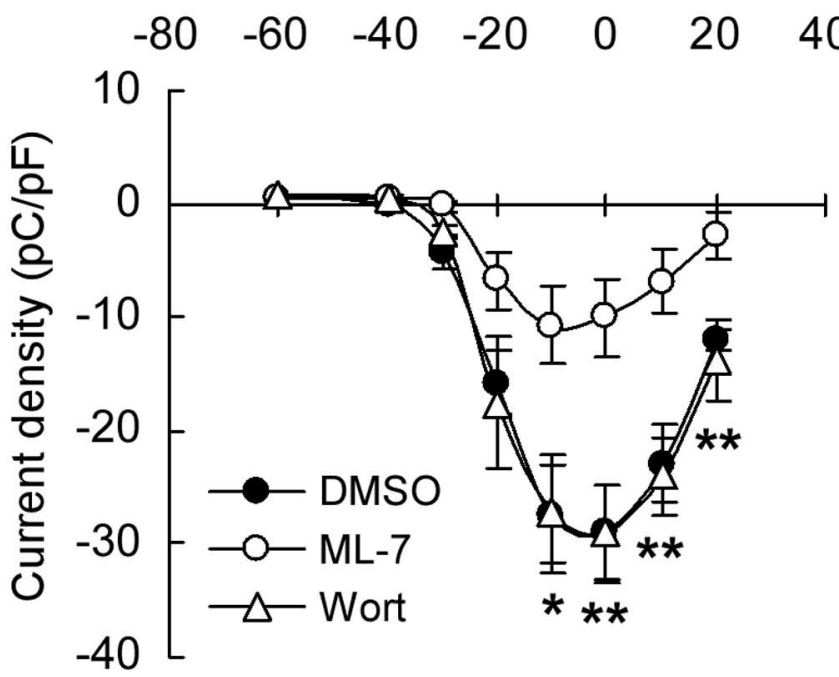

Figure 4. ML-7 reduces VGCC current. $A$, Overlay of representative VGCC current traces induced by a $30 \mathrm{~ms}$ voltage step to $0 \mathrm{mV}$ from $-80 \mathrm{mV}$ in neurons pretreated with $0.1 \%$ DMSO or $10 \mu \mathrm{M}$ ML-7. $\boldsymbol{B}$, Summary of VGCC current density induced by a voltage step to indicated membrane potentials in neurons pretreated with $0.1 \%$ DMSO $(n=9), 10 \mu \mathrm{M} \mathrm{ML-7}(n=10)$, or $10 \mu \mathrm{M}$ wortmannin $(n=7)$. Solid lines are cubic spline interpolation. ML-7 decreases VGCC currents relative to DMS0, whereas wortmannin has no effect $\left(^{*} p<0.05\right.$, ${ }^{* *} p<0.01$; Dunnett's test, DMSO vs ML-7). Error bars indicate SEM.

RRP. Calcimycin application for 2 min released on average $\sim 32$ $\mathrm{nC}$, whereas the RRP size in our autaptic system was estimated as $\sim 4 \mathrm{nC}$ (Fig. $2 I$, taking into account that $1 \mu \mathrm{M}$ CNQX was applied to reduce the charge transfer to $\sim 25 \%$ in sucrose experiments but not for monitoring calcimycin-triggered release). Therefore, calcimycin-dependent release must have accessed not only RRP vesicles but also vesicles from the reserve or the recycled pool; yet ML-7 had no effect on the total amount of release. Together, our data suggest that MLCK activity is not required for supplying synaptic vesicles needed for sustained release on elevation of intracellular $\mathrm{Ca}^{2+}$.

To study the refilling of the RRP under basal intracellular $\mathrm{Ca}^{2+}$ levels, we turned to RRP depletion by sucrose application, which, unlike calcimycin, can be used to trigger release repeatedly by a $\mathrm{Ca}^{2+}$-independent process (Rosenmund and Stevens, 1996). We tested the extent of RRP recovery by applying the second pulse of sucrose at $3.6 \mathrm{~s}$ after the first pulse, at a time when RRP was expected to recover to about one-half of its full RRP size (Morales et al., 2000). We found no significant effect of ML-7 nor of wortmannin on the extent of RRP replenishment (control
DMSO, $0.54 \pm 0.08$; ML-7, $0.57 \pm 0.07$; wortmannin, $0.53 \pm$ 0.08; one-way ANOVA, $p>0.9$ ) (Fig. 5D). Moreover, there were no significant dose-dependent effects of ML-7 and wortmannin when tested up to $30 \mu \mathrm{M}$ (supplemental Fig. 5, available at www. jneurosci.org as supplemental material). Thus MLCK is not likely to play an essential role for RRP replenishment under basal conditions.

\section{Wortmannin does not change the rate of evoked unloading of FM1-43}

To further explore the involvement of MLCK in fast repetitive release, we compared the effects of ML-7 and wortmannin on SV exocytosis monitored by the unloading kinetics of FM1-43, after its endocytic uptake into recycling synaptic vesicles. Because wortmannin may affect endocytosis (Richards et al., 2004) and, in turn, complicate the interpretation of exocytic FM dye loss, SVs were first loaded with FM1-43 by $10 \mathrm{~Hz}$ field stimulation in the absence of the drug. Neurons were subsequently treated with control DMSO, $10 \mu \mathrm{M}$ ML-7, or $10 \mu \mathrm{M}$ wortmannin for $15 \mathrm{~min}$, and then stimulated by $20 \mathrm{~Hz}$ field stimulation to evoke FM1-43 unloading (Fig. 6A). As reported previously and expected from the effects on APs and VGCC (Figs. 3, 4), ML-7 significantly slowed the rate of dye loss compared with control neurons treated with DMSO (Fig. 6B,C). However, wortmannin had no effect even beyond the first few seconds of stimulation (Fig. 6B, C) when vesicle exocytosis is balanced by recruitment of vesicles from the reserve or the recycling pool (Fig. 2D). Both curves were well fit by a monoexponential decay function with similar time constants (DMSO, $13.0 \pm 0.5 \mathrm{~s}$; wortmannin, $13.7 \pm 0.6 \mathrm{~s} ; t$ test, $p>0.4)$ and resulted in comparable level of the residual fluorescence at the end of the stimulus train (DMSO, $29.0 \pm 0.1 \%$; wortmannin, $29.0 \pm 0.1 \%$; $t$ test, $p>0.9$ ). Collectively, these data indicate that MLCK activity is not essential for fast repetitive exocytosis.

\section{Discussion}

\section{Specificity and action of MLCK inhibitors}

ML-7 and ML-9 are potent chemical inhibitors that target the catalytic site of MLCK (Saitoh et al., 1987), and they display higher specificity than wortmannin toward MLCK (Arcaro et al., 1992; Nakanishi et al., 1992; Yano et al., 1995). Consequently, ML-7 and ML-9 have been popularly used for studying MLCK function in various cell types. Characterization of these inhibitors in neurons, however, has been very limited. In our attempt to clarify the role for MLCK in regulating synaptic vesicle traffic by pharmacological manipulation, we unexpectedly found that ML-7 and ML-9 exerted nonspecific actions in neurons. Consistent with the proposed role for MLCK in facilitating neurotransmitter release, these drugs enhanced short-term depression and reduced presynaptic release probability. However, the two inhibitors also attenuated APs and the VGCC current and increased the frequency of spontaneous neurotransmitter release. In contrast, wortmannin, at a concentration effective for blocking MLCK, did not mimic these effects of ML-7 and ML-9 on neuronal excitability and spontaneous neurotransmitter release.

The observed reduction in presynaptic release probability and enhanced short-term depression in the presence of ML-7 are expected from the compromised APs and the VGCC current. The inhibition of voltage-gated sodium channels could account for the reduced AP amplitude and the increase in short-term depression (Brody and Yue, 2000). Furthermore, altered activity of potassium channels could contribute to the broadening of the AP waveform (Kim et al., 2005). Whereas the spike broadening itself 
is not fully compatible with reduced neurotransmitter release, inhibition of VGCC at the nerve terminal would decrease release probability by limiting the excitation-coupled rise in intraterminal $\mathrm{Ca}^{2+}$. Although we monitored the effects of ML-7 and ML-9 on somatic VGCC currents in this study, the nonspecific actions of these MLCK inhibitors might be exerted similarly on VGCC at the presynaptic boutons. The inhibition of VGCC appeared to be nonselective for channel subtypes because in some neurons $\mathrm{Ba}^{2+}$ currents were completely blocked by either ML-7 (data not shown) or ML-9 (supplemental Fig. 4, available at www.jneurosci.org as supplemental material). Our data do not explain whether various voltage-gated channels are the direct targets of ML-7 and ML-9. Modulation of neuronal excitability could result from affecting the activities of other membrane and intracellular protein targets. Collectively, the direct contribution of MLCK inhibition by ML-7 and ML- 9 on synaptic vesicle recruitment and exocytosis is difficult to assess under conditions in which neuronal excitability and VGCC activity are nonspecifically compromised by the inhibitors.

\section{Role of MLCK in presynaptic release}

Three lines of evidence support the conclusion that MLCK inhibition has no effect on the repetitive release of neurotransmitters at the presynaptic terminals. First, calcimycin directly activates the release machinery downstream of $\mathrm{Ca}^{2+}$ influx (Fernandez-Chacon et al., 2001; Reim et al., 2001), and consequently, neurotransmitter release induced by the $\mathrm{Ca}^{2+}$ ionophore should bypass the nonspecific inhibitory effects of ML-7 on APs and VGCCs. A decrease in calcimycin-induced release in the presence of ML-7, therefore, would indicate a requirement for MLCK in exocytosis. Moreover, because calcimycin triggers prolonged release of multiple rounds of RRP, if MLCK is needed for accessing the reserve vesicles to refill the RRP and maintain release, calcimycin-induced response should decline more rapidly in the presence of ML-7. However, neither the rate nor the amount of calcimycin-induced release was affected by ML-7. Second, to determine whether MLCK could be active at resting $\left[\mathrm{Ca}^{2+}\right]_{\mathrm{i}}$ levels to regulate SV trafficking, RRP replenishment was monitored by paired puffs of hypertonic sucrose solution to trigger exocytosis independently of extracellular $\mathrm{Ca}^{2+}$ (Rosenmund and Stevens, 1996). ML-7 had no effect on the rate of RRP refilling, suggesting that MLCK was not important even for basal SV trafficking at resting $\left[\mathrm{Ca}^{2+}\right]_{\mathrm{i}}$. Third, we examined the effect of wortmannin on the kinetics of FM1-43 dye loss from the total recycling pool vesicles that were loaded in the absence of the drug (900 APs at $10 \mathrm{~Hz}$ ) (Ryan and Smith, 1995). The kinetics of dye loss, which should be driven by the exocytosis of RRP and recycling pool vesicles, was not altered in the presence of wortmannin. Thus, these findings lead us to conclude that MLCK is not a key regulator of SV trafficking during repetitive exocytosis.

Previously, at least two reports proposed the involvement of
MLCK in presynaptic function at hippocampal synapses. ML-7 and ML-9 were found to slow the kinetics of FM1-43 dye unloading induced by repetitive field stimulation but not the release from RRP, which suggested a decrease in mobilization of the reserve pool (Ryan, 1997). However, based on our data, such slowing of the rate of FM1-43 unloading was likely caused by inhibiting neuronal firing by ML-7 and ML-9. Moreover, an apparent lack of change on RRP exocytosis observed in this previous study could be attributable to a relatively short time of drug treatment (150 s) or compensation by the large increase in spontaneous exocytosis that we found in the present study. In another report, ML-7 treatment dramatically reduced SV motility, as measured by fluorescence fluctuation spectroscopy of FM1-43labeled vesicles (Jordan et al., 2005). Despite the decreased SV motility, ML-7 did not affect calcimycin-induced release nor refilling of RRP depleted by sucrose. Therefore, processes underlying general vesicle motility and targeted vesicle recruitment to the releasable pool might be uncoupled and regulated differentially.

We also determined the subcellular localization of MLCK by immunocytochemistry. Potential regulation of SV mobilization by MLCK would warrant its presence at the presynaptic SV cluster. However, the MLCK signal did not highly colocalize with a vesicle marker, consistent with our main electrophysiological findings of the lack of participation of MLCK in regulating SV recruitment for exocytosis. Instead, a strong signal was observed throughout the axon, suggestive of a major role for MLCK in axonal transport between boutons. 


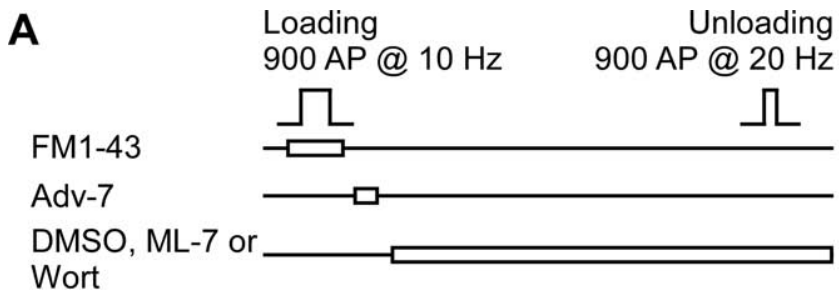

B
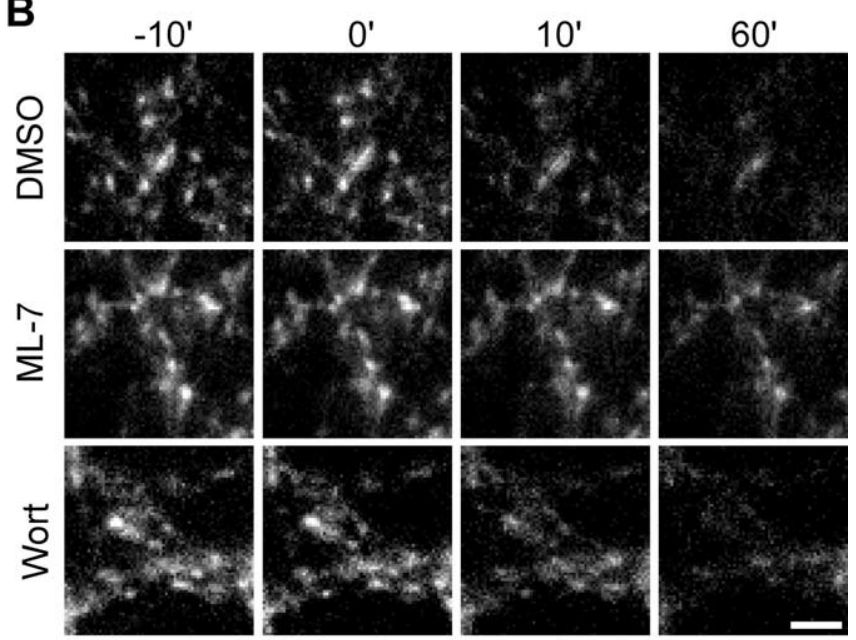

C

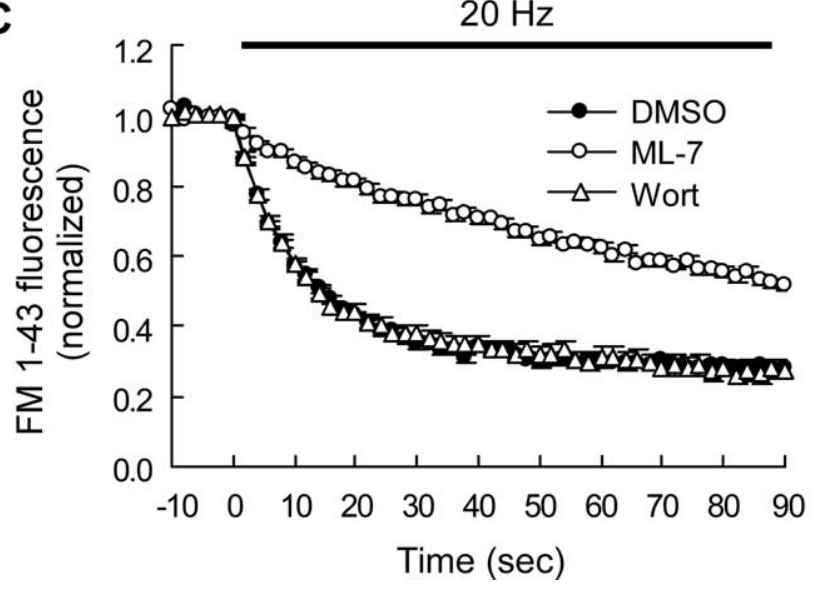

Figure 6. Wortmannin does not inhibit FM1-43 unloading rate. $\boldsymbol{A}$, Experimental scheme of FM1-43 loading, drug treatment, and FM1-43 unloading by field stimulation. Wort, Wortmannin; Adv-7, Advasep-7. B, Representative images of FM1-43 in neurons treated with $0.1 \%$ DMSO, $10 \mu \mathrm{M} \mathrm{ML-7,} \mathrm{or} 10 \mu \mathrm{m}$ wortmannin at indicated times corresponding to the start of the unloading stimulus train. Scale bar, $4 \mu \mathrm{m}$. C, Summary of the time course of FM1-43 unloading in neurons treated with DMSO ( $n=80$ puncta, 4 coverslips), $10 \mu \mathrm{M} \mathrm{ML-7} \mathrm{(} n=$ 60 puncta, 3 coverslips), or $10 \mu \mathrm{m}$ wortmannin ( $n=80$ puncta, 4 coverslips). Data were normalized to the average FM1-43 fluorescence of the first six frames captured before the unloading stimulus. Error bars indicate SEM.

Role of myosin-actin system in presynaptic vesicle trafficking Given that MLCK does not appear to play a key role in SV mobilization, is myosin-actin system used for trafficking of SVs to the RRP at the presynaptic boutons? Notably, ATP is not required for SV replenishment in cultured hippocampal neurons (Mozhayeva et al., 2004), implying that myosin ATPase activity may be dispensable. Several studies to date have focused on the role of actin filament dynamics at hippocampal synapses. In mature synapses, depolymerizing F-actin with latrunculin A or B had no effect on RRP recovery rate (Morales et al., 2000), total recycling pool size
(Zhang and Benson, 2001), rates of SV endocytosis and repriming (Sankaranarayanan et al., 2003), and SV motility (Jordan et al., 2005). Moreover, preventing F-actin turnover with jasplakinolide did not change the kinetics of SV exocytosis (Sankaranarayanan et al., 2003) and short-term depression of EPSCs (our unpublished observation). Collectively, these data point to a nonessential role for actin filaments and their turnover for SV trafficking, and raise the possibility that perhaps myosin-actin system is not necessary for sustaining the repeated rounds of exocytosis. However, because the principal target of MLCK is myosin II, our data do not exclude a role for other myosin family members present in neurons, including myosin I, V, VI, and IX (Bridgman, 2004). Interestingly, loss of myosin VI was recently demonstrated to reduce the number of functional synapses and produce defects in evoked presynaptic release in hippocampal neurons (Yano et al., 2006). Whether a role for myosin VI in SV traffic in the presynaptic boutons is independent of its function in synapse development and/or stability remains to be clarified.

Although we find that actin and MLCK are not essential for SV recruitment to the RRP in hippocampal synapses, actin, myosin, and MLCK have been implicated in this process in other types of neurons. For example, in Drosophila neuromuscular junctions, mobilization of reserve pool vesicles is regulated by actin dynamics (Kuromi and Kidokoro, 1998; Delgado et al., 2000) and is propelled by myosin that is fueled by the mitochondrial ATP (Verstreken et al., 2005). At mouse neuromuscular junctions, MLCK is essential for maintaining transmission during repetitive stimulation (Polo-Parada et al., 2005), and in calyx of Held synapses, actin filaments promote SV replenishment to the releasable pool (Sakaba and Neher, 2003). Thus, in these big synapses, myosin-actin system appears to be important for efficient trafficking of SVs. In contrast, in small synapses of CNS neurons, vesicles may be recruited by an actin-independent mechanism, for instance, by passive diffusion. Notably, in cone photoreceptor terminals and retinal bipolar cells in which continuous exocytosis occurs, diffusion appears to be the main mechanism of SV trafficking (Job and Lagnado, 1998; Holt et al., 2004; Rea et al., 2004). Therefore, diffusion could be fast enough to sustain repeated cycles of SV exocytosis in small CNS synapses as well. Accordingly, SV mobility does not change during synaptic activity compared with the resting state (Lemke and Klingauf, 2005). Nonetheless, modulation of protein-protein interactions by various signaling pathways could alter the properties of vesicle diffusion with consequences on SV mobilization (Chi et al., 2003). Uncovering regulatory mechanisms of SV mobilization in connection with anchoring and docking should help delineate how the replenishment of SVs influences the repetitive triggering of exocytosis that is central to the induction of short-term plasticity.

\section{References}

Arcaro A, Wymann MP (1993) Wartmannin is a potent phosphatidylinositol 3-kinase inhibitor: the role of phosphatidylinositol 3,4,5-triphosphate in neutrophil responses. Biochem J 296:297-301.

Bekkers JM, Stevens CF (1991) Excitatory and inhibitory autaptic currents in isolated hippocampal neurons maintained in cell culture. Proc Natl Acad Sci USA 88:7834-7838

Benson DL, Mandell JW, Shaw G, Banker G (1996) Compartmentation of alpha-internexin and neurofilament triplet proteins in cultured hippocampal neurons. J Neurocytol 25:181-196.

Berg JS, Powell BC, Cheney RE (2001) A millennial myosin census. Mol Biol Cell 12:780-794.

Bridgman PC (2004) Myosin-dependent transport in neurons. J Neurobiol 58:164-174.

Brody DL, Yue DT (2000) Release-independent short-term synaptic depression in cultured hippocampal neurons. J Neurosci 20:2480-2494. 
Chi P, Greengard P, Ryan TA (2003) Synaptic vesicle mobilization is regulated by distinct synapsin I phosphorylation pathways at different frequencies. Neuron 38:69-78.

De La Cruz EM, Ostap EM (2004) Relating biochemistry and function in the myosin superfamily. Curr Opin Cell Biol 16:61-67.

Delgado R, Maureira C, Oliva C, Kidokoro Y, Labarca P (2000) Size of vesicle pools, rates of mobilization, and recycling at neuromuscular synapses of a Drosophila mutant, shibire. Neuron 28:941-953.

Drenckhahn D, Kaiser HW (1983) Evidence for the concentration of F-actin and myosin in synapses and in the plasmalemmal zone of axons. Eur J Cell Biol 31:235-240.

Dunaevsky A, Connor EA (2000) F-actin is concentrated in nonrelease domains at frog neuromuscular junctions. J Neurosci 20:6007-6012.

Fernandez-Chacon R, Konigstorfer A, Gerber SH, Garcia J, Matos MF, Stevens CF, Brose N, Rizo J, Rosenmund C, Sudhof TC (2001) Synaptotagmin I functions as a calcium regulator of release probability. Nature 410:41-49.

Grimm M, Haas P, Willipinski-Stapelfeldt B, Zimmermann WH, Rau T, Pantel K, Weyand M, Eschenhagen T (2005) Key role of myosin light chain (MLC) kinase-mediated MLC2a phosphorylation in the alpha 1 -adrenergic positive inotropic effect in human atrium. Cardiovasc Res 65:211-220.

Hagler Jr DJ, Goda Y (2001) Properties of synchronous and asynchronous release during pulse train depression in cultured hippocampal neurons. J Neurophysiol 85:2324-2334.

Holt M, Cooke A, Neef A, Lagnado L (2004) High mobility of vesicles supports continuous exocytosis at a ribbon synapse. Curr Biol 14:173-183.

Job C, Lagnado L (1998) Calcium and protein kinase C regulate the actin cytoskeleton in the synaptic terminal of retinal bipolar cells. J Cell Biol 143:1661-1672.

Jordan R, Lemke EA, Klingauf J (2005) Visualization of synaptic vesicle movement in intact synaptic boutons using fluorescence fluctuation spectroscopy. Biophys J 89:2091-2102.

Kamm KE, Stull JT (2001) Dedicated myosin light chain kinases with diverse cellular functions. J Biol Chem 276:4527-4530.

Kim J, Wei DS, Hoffman DA (2005) Kv4 potassium channel subunits control action potential repolarization and frequency-dependent broadening in rat hippocampal CA1 pyramidal neurons. J Physiol (Lond) 569:41-57.

Kuromi H, Kidokoro Y (1998) Two distinct pools of synaptic vesicles in single presynaptic boutons in a temperature-sensitive Drosophila mutant, shibire. Neuron 20:917-925.

Langford GM (1995) Actin- and microtubule-dependent organelle motors: interrelationships between the two motility systems. Curr Opin Cell Biol $7: 82-88$.

Lemke EA, Klingauf J (2005) Single synaptic vesicle tracking in individual hippocampal boutons at rest and during synaptic activity. J Neurosci 25:11034-11044.

Mochida S, Nonomura Y, Kobayashi H (1994) Myosin II is involved in transmitter release at synapses formed between rat sympathetic neurons in culture. Neuron 13:1131-1142.

Morales M, Colicos MA, Goda Y (2000) Actin-dependent regulation of neurotransmitter release at central synapses. Neuron 27:539-550.

Mozhayeva MG, Matos MF, Liu X, Kavalali ET (2004) Minimum essential factors required for vesicle mobilization at hippocampal synapses. J Neurosci 24:1680-1688.

Nakanishi S, Kakita S, Takahashi I, Kawahara K, Tsukuda E, Sano T, Yamada K, Yoshida M, Kase H, Matsuda Y (1992) Wortmannin, a microbial product inhibitor of myosin light chain kinase. J Biol Chem 267:2157-2163.

Otsu Y, Shahrezaei V, Li B, Raymond LA, Delaney KR, Murphy TH (2004) Competition between phasic and asynchronous release for recovered synaptic vesicles at developing hippocampal autaptic synapses J Neurosci 24:4204-4233.
Polo-Parada L, Plattner F, Bose C, Landmesser LT (2005) NCAM 180 acting via a conserved C-terminal domain and MLCK is essential for effective transmission with repetitive stimulation. Neuron 46:917-931.

Qi D, Mitchell RW, Burdyga T, Ford LE, Kuo KH, Seow CY (2002) Myosin light chain phosphorylation facilitates in vivo myosin filament reassembly after mechanical perturbation. Am J Physiol 282:C1298-C1305.

Rea R, Li J, Dharia A, Levitan ES, Sterling P, Kramer RH (2004) Streamlined synaptic vesicle cycle in cone photoreceptor terminals. Neuron 41:755-766.

Reim K, Mansour M, Varoqueaux F, McMahon HT, Sudhof TC, Brose N, Rosenmund C (2001) Complexins regulate a late step in $\mathrm{Ca}^{2+}$. dependent neurotransmitter release. Cell 104:71-81.

Richards DA, Rizzoli SO, Betz WJ (2004) Effects of wortmannin and latrunculin A on slow endocytosis at the frog neuromuscular junction. J Physiol (Lond) 557:77-91.

Rosenmund C, Stevens CF (1996) Definition of the readily releasable pool of vesicles at hippocampal synapses. Neuron 16:1197-1207.

Ryan TA (1997) Inhibitors of myosin light chain kinase block synaptic vesicle pool mobilization during action potential firing. J Neurosci 19:1317-1323.

Ryan TA, Smith SJ (1995) Vesicle pool mobilization during action potential firing at hippocampal synapses. Neuron 14:983-989.

Saitoh M, Ishikawa T, Matsushima S, Naka M, Hidaka H (1987) Selective inhibition of catalytic activity of smooth muscle myosin light chain kinase. J Biol Chem 262:7796-7801.

Sakaba T, Neher E (2003) Involvement of actin polymerization in vesicle recruitment at the calyx of Held synapse. J Neurosci 3:837-846.

Sakurada K, Seto M, Sasaki Y (1998) Dynamics of myosin light chain phosphorylation at Ser19 and Thr18/Ser19 in smooth muscle cells in culture. Am J Physiol 274:C1563-C1572.

Sankaranarayanan S, Atluri PP, Ryan TA (2003) Actin has a molecular scaffolding, not propulsive, role in presynaptic function. Nat Neurosci 6:127-135.

Schnell E, Nicoll RA (2001) Hippocampal synaptic transmission and plasticity are preserved in myosin Va mutant mice. J Neurophysiol 85:1498-1501.

Stevens CF, Wesseling JF (1998) Activity-dependent modulation of the rate at which synaptic vesicles become available to undergo exocytosis. Neuron 21:415-424.

Takagishi Y, Futaki S, Itoh K, Espreafico EM, Murakami N, Murata Y, Mochida S (2005) Localization of myosin II and V isoforms in cultured rat sympathetic neurones and their potential involvement in presynaptic function. J. Physiol 569:195-208.

Takayama M, Ozaki H, Karaki H (1996) Effects of a myosin light chain kinase inhibitor, wortmannin, on cytoplasmic $\mathrm{Ca}^{2+}$ levels, myosin light chain phosphorylation and force in vascular smooth muscle. Naunyn Schmiedebergs Arch Pharmacol 354:120-127.

Verstreken P, Ly CV, Venken KJ, Koh TW, Zhou Y, Bellen HJ (2005) Synaptic mitochondria are critical for mobilization of reserve pool vesicles at Drosophila neuromuscular junctions. Neuron 47:365-378.

$\mathrm{Xu} \mathrm{J,} \mathrm{Wu} \mathrm{LG} \mathrm{(2005)} \mathrm{The} \mathrm{decrease} \mathrm{in} \mathrm{the} \mathrm{presynaptic} \mathrm{calcium} \mathrm{current} \mathrm{is} \mathrm{a}$ major cause of short-term depression at a calyx-type synapse. Neuron 46:633-645.

Yano H, Agatsuma T, Nakanishi S, Saitoh Y, Fukui Y, Nonomura Y, Matsuda Y (1995) Biochemical and pharmacological studies with KT7692 and LY294002 on the role of phosphatidylinositol 3-kinase in FceRI-mediated signal transduction. Biochem J 312:145-150.

Yano H, Ninan I, Zhang H, Milner TA, Arancio O, Chao MV (2006) BDNFmediated neurotransmission relies upon a myosin VI motor complex. Nat Neurosci 9:1009-1018.

Zhang W, Benson DL (2001) Stages of synapse development defined by dependence on F-actin. J Neurosci 21:5169-5181. 